\section{TECHNIQUES AND APPLICATIONS}

\section{Reverse genetics for Borna disease virus}

Two papers have described a reverse genetics system for Borna disease virus (BDV), a non-cytolytic neurotropic virus that has been connected with neuropsychiatric disorders in humans.

Investigation of the molecular basis for BDV persistence in the central nervous system has been hampered by the lack of a genetic system to study its genome and gene products. BDV is a non-segmented, enveloped virus with an 8.9-kb negativestrand RNA genome. Like all negative-strand RNA viruses, the minimal infectious unit of $\mathrm{BDV}$ is a ribonucleoprotein complex comprising the viral RNA genome tightly associated with viral proteins.

In the strategy described by Perez et al., a BDV minigenome was constructed that comprised a reporter gene flanked upstream by the $5^{\prime}$ untranslated region (UTR) of the $\mathrm{BDV}$ genome and a murine RNA polymerase I promoter, and downstream by the BDV $3^{\prime}$ UTR and the murine RNA polymerase I terminator sequence. In the strategy described by Schneider et al., the reporter gene was flanked upstream by the BDV $5^{\prime}$ UTR and the $\mathrm{T} 7$ promoter, and transcription termination downstream was controlled by the hepatitis delta virus ribozyme.

The roles of trans-acting BDV proteins in transcription of the BDV genome were investigated by co-transfecting cells with plasmid vectors encoding these proteins under control of the chicken $\beta$-actin promoter or a modified version. Both groups found that the stoichiometry of the ratio of $\mathrm{BDV}$ nucleocapsid $(\mathrm{N})$ protein to phosphoprotein $(\mathrm{P})$ was a key factor in the regulation of the $\mathrm{BDV}$ polymerase complex. In addition, both reports documented that the BDV X protein (also called p10) is a negative regulator of the viral polymerase. Genomic manipulations of the negativestrand RNA viruses have lagged behind that of viruses with either DNA or positivestrand RNA genomes. However, progress has been made in the past decade and reverse genetics systems are now available

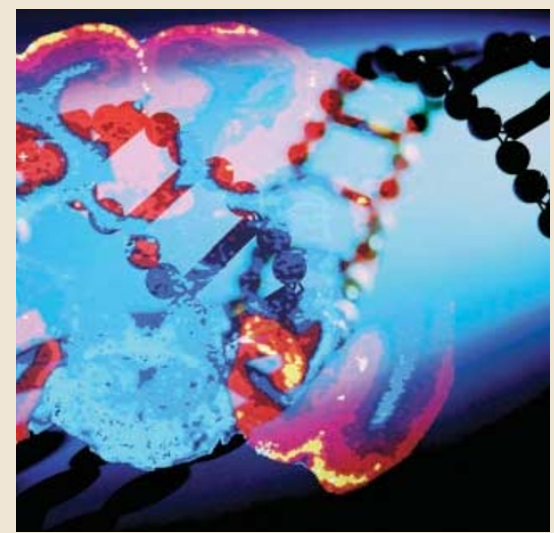

for several negative-strand RNA viruses. The development of these analytical tools for BDV will allow the investigation of both the cis-acting signals and trans-acting factors that are involved in transcription and replication of the BDV genome. Sheilagh Clarkson

\section{0) References}

ORIGINAL RESEARCH PAPERS Perez, M. et al

A reverse genetics system for Borna disease virus. J. Gen. Virol. 84, 3099-3104 (2003) | Schneider, S. et al. Active Borna disease virus polymerase complex requires a distinct nucleoprotein-to-phosphoprotein ratio but no viral $X$ protein J. Virol. 77, 11781-11789 (2003)

\title{
Attack thy neighbour
}

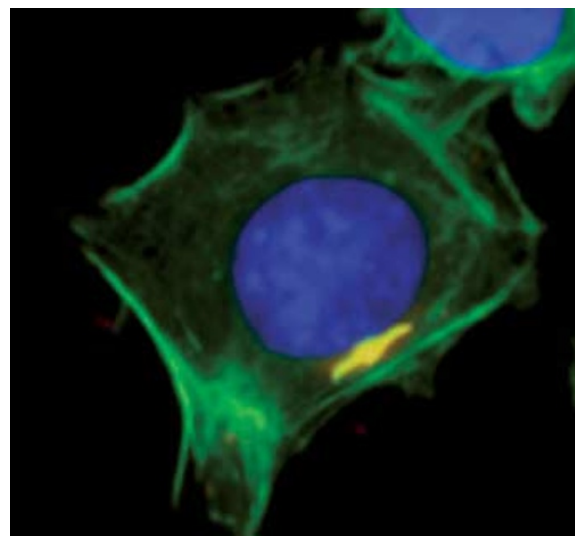

Image, kindly provided by M. Mota, shows actin recruitment around $P$. bergheii sporozoites in HepG2 cells (actin and parasite stained green and red, respectively. The parasite appears yellow owing to the merging of images).

The passage of Plasmodium parasites through liver cells stimulates the release of a host growth factor that increases the chances of neighbouring cells becoming infected, according to a report published in Nature Medicine.

The life cycle of Plasmodium spp., the parasite responsible for malaria, is divided between humans and mosquitoes. Humans become infected when they are bitten by infected mosquitoes - the sporozoite form of the parasite is transferred from the mosquito salivary glands into the host. The sporozoites are then carried in the bloodstream to the liver, where they establish an infection by invading and replicating within hepatocytes.

Malaria sporozoites do not invade the first hepatocyte they encounter - instead, they pass through and injure several cells by disrupting the plasma membrane before invasion of an hepatocyte occurs through formation of a specialized vacuole. Carrolo et al. hypothesized that the injured hepatocytes might release a factor that increases the susceptibility of neighbouring cells to sporozoite infection. They confirmed the existence of such a factor by incubating hepatocyte cells in media that had been pre-treated with Plasmodium yoelii sporozoites. In these cells, the level of infection seen during subsequent incubation with $P$. yoelii sporozoites was increased, whereas in cells incubated in media that had been pre-treated with heat-inactivated sporozoites, there was no increased susceptibility to infection.
Carrolo et al. postulated that the factor responsible for this increased susceptibility to infection could be one of the human growth factors known to be released from injured cells. In vivo assays showed that preincubation of hepatocytes with human hepatocyte growth factor (HGF) increased their susceptibility to infection with sporozoites. The identity of HGF as the factor responsible was confirmed by the fact that incubation with a neutralizing antibody to HFG blocked this increased susceptibility to infection.

HGF mediates its effects on host cells through the MET tyrosine kinase receptor. The involvement of MET in sporozoite infectivity was established through a variety of procedures, including experiments showing that sporozoite infectivity was enhanced by transfection of a constitutively active MET receptor and that downregulating MET activity decreases the rate of sporozoite infection. Further work revealed that the hepatocyte response to sporozoite-induced HGF/MET signalling involved reorganization of the actin cytoskeleton.

Sheilagh Clarkson

\section{(4) References and links}

ORIGINAL RESEARCH PAPER Carrolo, M. et al. Hepatocyte growth factor and its receptor are required for malaria infection. Nature Med. 12 October 2003 (doi: 10.1038/nm947) WEB SITE

Nature Focus on malaria:

http://www.nature.com/nature/malaria/ 